\title{
Ectomycorrhizal Diversity in Zabarvan Forest Range of North Western Himalaya
}

\author{
P.A. Sheikh ${ }^{1}$, Baby Summuna ${ }^{1}$, G.H. Dar ${ }^{1}$, Sajad-Un-Nabi ${ }^{2}$ and Khurshid Ahmad Mir ${ }^{3}$ \\ ${ }^{1}$ Division of Plant Pathology, S.K. University of Agricultural Sciences \& Technology of \\ Kashmir, Shalimar, Srinagar-190 025 Jammu and Kashmir, India \\ ${ }^{2}$ Central Institute of Temperate Horticulture, Indian council of Agriculture Research \\ Srinagar-190007 Jammu and Kashmir, India \\ ${ }^{3}$ Division of Vegetable science, S.K. University of Agricultural Sciences \& Technology of \\ Kashmir, Shalimar, Srinagar-190 025 Jammu and Kashmir, India
}

*Corresponding author

\section{Keywords \\ Biodiversity index, Ectomycorrhiza, Kashmir \\ Himalayas, Species Richness, Zabarvan forest \\ Article Info \\ Accepted: 18 April 2019 Available Online: 10 May 2019}

\section{A B S T R A C T}

The aim of the present investigation was to prepare an inventory of ectomycorrhiza prevailing in Zabarvan forest range of Western Himalayas of Kashmir and assess their diversity index and species richness. Three forest sites viz., Dachigam, Shalimar and Shankaracharia hills were surveyed periodically for three consecutive years (2011-2013) for ectomycorrhiza. A total number of 67 species in 23 genera belonging to 21 families in 07 orders were recorded. The study revealed that mycorrhizal fungal species richness was more in autumn season (45 species) and less in summer (11 species). The Simpson diversity index of Dachigam, Shalimar and Shankaracharia hills was found to be 0.981, 0.910, 0.939 respectively, while Shannon's diversity index of these sites was 4.03, 2.36, 2.903 , respectively.

\section{Introduction}

Biological diversity is a central determinant of ecosystem function and also a key contributor to the portfolio of services provided by ecosystems to humans (Carlson et al., 2007). India, one among 12 mega diversity countries across the globe, possesses more than $8 \%$ of the world's total biodiversity and its bio-geographic ecosystems is classified into ten zones which include Trans-
Himalaya and Himalaya zones (Rodgers et al., 2002). Mapping biological diversity of a region is a major goal to the global conservation community (Gaston, 2000). Forest and tree cover of India constitutes nearly $789,164 \mathrm{~km}^{2}$, which constitute $24 \%$ of geographical area of the country (FSI, 2013). The country's rich vegetation and diversity is undoubtedly due to the immense variety of climatic and altitudinal variations (Reddy et al., 2013). The state of Jammu and Kashmir 
lies in the North Western Himalayan mountainous range between $32^{\circ} 17^{\prime}$ and $37^{\circ} 05^{\prime}$ North latitude and $72^{\circ} 31^{\prime}$ and $80^{\circ} 20^{\prime}$ East longitude with geographic area of $101,387 \mathrm{~km}^{2}$ of which $19.95 \%$ area is covered by forests alone (ES, 2013-14). Of the total forest area in $\mathrm{J} \& \mathrm{~K}$ state, $40.2 \%$ area lies in Kashmir valley alone which harbours rich floristic diversity.

As per the conservative estimates, about 1.5 million fungal species are present worldwide (Hawksworth, 2004) with one-third existing in India alone. Hardly $50 \%$ of these fungi have been identified and characterized so far (Manoharachary et al., 2005). Ectomycorrhizal plants, while taxonomically more rare, are common within boreal and temperate forests (e.g. Pinaceae, Fagaceae, Betulaceae, Nothofagaceae and others) (Tedersoo and Smith, 2013).

The floristic composition of North West Himalayan mountains, have not extensively been surveyed and explored for the macrofungi emanating in different seasons, except for some widely used edible mushrooms (Kaul et al., 1978; Walting and Abraham, 1992; Samant and Dhar, 1997). Hardly 250 macrofungi species have so far been reported from the $\mathrm{J} \& \mathrm{~K}$ (Walting and Abraham, 1992; Beig et al., 2008; Dar et al., 2009; Sheikh et al., 2014), mostly from Gulmarg and Pahalgam forests. The Zabarvan forest range which harbours mixed- and pureconifer stands and stretches from Shankaracharia hills to Dachigham hills has not been extensively surveyed for mycotic flora so far. The baseline information on diversity in different vegetation types is essential for planning and managing ecosystem biodiversity (Engola et al., 2007). The knowledge about biodiversity at community and species levels is important for monitoring the effectiveness and impact of natural and artificial disturbances (Packham et al., 2002). Ectomycorhiza play important role in rendering the unavailable organic forms of soil nutrients available to the plants through various mechanisms including the production of extracellular enzymes (Read and PerezMoreno, 2003; Aucina et al., 2007). Enormous interest has recently generated in the use of ectomycorrhiza as inocula for successful forest nursery raising especially in degraded and degenerated forests. The present study was therefore, aimed to assess the mycorrhizal diversity of Zabarvan forest range in Kashmir Himalayas, prepare inventory and assess their diversity index and species richness.

\section{Materials and Methods}

\section{Collection site}

The Zabarvan forest range (Fig. 1) lies in the north of Srinagar city of Jammu \& Kashmir State which lies in the heart of North Western Himalaya. It lies at $34^{\circ} 02$ and $34^{\circ} 08 \mathrm{~N}$ latitude and $74^{\circ} 44$ and $74^{\circ} 55 \mathrm{E}$ longitude and covers an area of $265 \mathrm{~km}^{2}$. The sites selected for macrofungal survey were Dachigam, Shalimar and Shankaracharia hills which cover an area of 141, 69 and $55 \mathrm{~km}^{2}$, respectively, with altitude ranging from 16764267, 1624-3385 and 1585-3352 $\mathrm{m}$ masl, respectively. These forests are mostly dominated by conifers viz., cedar, pine, spruce, fir, etc. growing upto $3657 \mathrm{~m}$ masl and above this altitude lie meadows which bloom with rhododendrons, honey suckle and dwarf willows.

The vegetative cover existing in these forests provide best suited habitat for fungal flora. The area has temperate climate with average temperature of $13.5^{\circ} \mathrm{C}$, the highest monthly average temperature of $17.3^{\circ} \mathrm{C}, 29.3^{\circ} \mathrm{C}$ and $25.5^{\circ} \mathrm{C}$ in March-April, June-July and September-October, respectively. The average rainfall is $710 \mathrm{~mm}$. 


\section{Sporocarp survey and identification}

The survey for the collection of ectomycorrhizal was carried out at monthly interval from March onwards during the years 2011 and 2013 in each potential growing season viz., spring, summer and fall (autumn). The efforts were made to establish the relation/association of sporocarps with plant root by careful digging of soil and tracing their connection with the host plant roots as per the method of Young (1940) and Zak (1969). The epigeous ectomycorrhiza of each fungal species were collected and wrapped in thin aluminum foil paper placed in polybags separately, brought to the laboratory and analyzed for their identity. Photographs were taken using digital Sony camera DSC-RX100. The spore prints were taken on paper or glass slides to study the colour of spores, shape of gills and pores, and attachment of gills to the stipe (Kuo, 2001; Kuo, 2004). The colour terminology followed was that of Kornerup and Wanscher (1978). Melzer's reagent was used to investigate amyloidity of pores and various other tissues. Cresyl blue solution was used to study the meta-chromatic reactions of spores. Specimens were identified on comparison with relevant literature (Kirk et al., 2001; de Roman et al., 2005; Agerer, 2006) and the information available at various web resources viz., Determination of Ectomycorrhiza (DEEMY), http://www. deemy.de; www.Mushroom Expert.com; mycokey, www.mycokey.com; Mycorrhiza literature exchange, http://mycorrhiza.ag.utk. $e d u$, etc.. The sporocarps were preserved in the herbarium of Mycology and Forestry Section, Division of Plant Pathology, SKUAST-Kashmir, Shalimar, Srinagar $(\mathrm{J} \& \mathrm{~K})$.

\section{Data analysis}

Simpson's diversity index was assessed as per Simpson (1949)
$D=\frac{\sum n(n-1)}{N(N-1)}$

$\mathrm{D}=$ Simpson's index, $\mathrm{N}=$ the total number of organisms of all species; $\mathrm{n}=$ the total number of organisms of a particular species

Shannon's diversity index was estimated as per Margalef (2008).

$H=-\sum\left(\frac{n}{N}\right) \log _{\varepsilon}\left(\frac{n}{N}\right)$

Where, $\mathrm{H}=$ the diversity index, $\mathrm{N}=$ the total number of individuals of all species; $\mathrm{n}=$ the total number of individuals of the individual species

With the help of the values of diversity index, the evenness of ectomycorrhiza was calculated as per Pielou (1996)

$\mathrm{e}=\mathrm{H} / \log _{\mathrm{e}} \mathrm{S}$

$\mathrm{E}=$ evenness, $\mathrm{H}=$ Shannon diversity index; $\mathrm{S}$ $=$ the number of the species

Similarity of index was estimated as per Sorenson's formula to assess the similarity in species occurrence (Odum, 1971). The similarity ranges from 0 to 1 ( 1 indicates very similar, 0 indicates no similarity)

$S=2 C /(A+B)$

Wherein $\mathrm{S}$ is the degree of similarity, $\mathrm{A}$ and $\mathrm{B}$ are the number of the species at two different sites and $\mathrm{C}$ is number of species common to both collections.

\section{Results and Discussion}

During three year survey of Zabarvan forest hills in Kashmir, sixty seven ectomycorrhizal species were collected from different locations and seasons (Table 1). These 
macrofungi were collected at an altitudinal range of 1825 to $2896 \mathrm{~m}$ masl. The hill range has predominantly coniferous forest stands either pure or mixed with broad-leaved plant species which support rich macrofungal flora. Conifer habitat provides congenial conditions for growth and sporulation of diverse macrofungi. Vishwakarma (2010) and Vishwakarma et al., (2011) have also noticed the presence of diverse macrofungi in conifer forests in Western Himalayas of Himachal Pradesh (India). These ectomycorrhiza have been collected from other forest ranges in Kashmir and described (Cooke, 1870; Murrill, 1924; Batra and Batra, 1963; Kaul and Kachroo, 1974; Watling and Gregory, 1980).

During survey, the ectomycorrhiza production was observed to be $16 \%$ higher in year 2012 and 2013 than year 2011. Higher sporocarp production in 2012 and 2013 may be ascribed to the favourable agro-climatic conditions especially higher and timely precipitation and congenial temperature from April to October. The year 2011 with mean precipitation of 210 $\mathrm{mm}$ and mean temperature of $24.2^{\circ} \mathrm{C}$ was comparatively drier and slightly warmer than 2012 and 2013 which probably may have affected the species diversity and ectomycorrhiza production by individual ectomycorrhiza species. The findings are in agreement with Mihali (1995) who during two year study in 1992 and 1993 observed 83 macromycetous species that produced 817 fruiting bodies in beech stand at Jalna, Slovak Republic.

The seasonal distribution of ectomycorrhiza across the Zabarvan forest range varied significantly with maximum ectomycorrhizal species witnessed in Dachigam followed by Shankaracharia and least in Shalimar hills. These variations may be attributed to varied latitude, vegetation, topography, etc. and their effects on temperature and precipitation across the wide geographic distances or along the elevational gradients. These findings are in conformity with Wood-Eggenschwiler and Barlocher (1985) and Ohenoja (1993) (Table 2).

A total number of 67 species in 23 genera of 21 families belonging to 05 orders of Basidiomycotina and 02 orders of Ascomycotina were considered for the ecological studies. They were identified up to species level. Agaricales dominated by $39 \%$ ( 8 families and 26 species in 09 genera) $25 \%$ of Boletales (07 families, 08 genera and 17 species) $21 \%$ Russulales (01 family, 02 genera and 14 species) Gomphales (01 family, 01 genera and 06 species) Thelephorales (02 families, 02 genera and 02 species) Mytilinidales (01 family, 01 genera and 01 species) Pezizales (01 family 01 genera and 01 species). Out of 21 families, Russulaceae dominated by $21 \%$ this is followed by Tricholomataceae 11\%, 9\% Gomphaceae and $7 \%$ Boletaceae, Inocybaceae and Suillaceae each. A list of ectomycorrhiza species family wise has been provided in Table 3 .

Ectomycorrhiza collected in three collection sites of Zabarvan forest range namely Dachigam, Shalimar and Shankaracharia were analyzed for ectomycorrhizal richness. The number of species collected area-wise showed maximum 65 species in Dachigam, 25 species in Shankaracharia and 11 species in Shalimar. Species diversity, richness and evenness of ectomycorrhiza in zabarvan range is shown in Table 4.

Out of 62 species collected from Dachigam, Agaricus xanthoderma, Boletus aereus, $B$. cavipes, B. gigas, Cenoccoum geophyllum, Chroomphogus tomentosus, C. vinicolor, Hebeloma crustuliniforme, H. cylindorosum, Hydnellum aurantiacum, Lycoperdon pedicillatum, Paxillus involutus, Pisolithus tinctorius, Ramaria aurea, $R$. flava, $R$. formosa, $R$. invalli, Rhizopogan roseulus, $R$. 
vulgaris, Russula atropurpurea, R. brevipes, $R$. delica, $R$. densifolia, $R$. emetica, $R$. lilacea, $R$. lutea, $R$. paludosa, $R$. sanguine, $R$. xerampelina, Russula sp., Scleroderma verrucosum, Suillus cavipes, S. granulates, $S$. luteus, S. placidus, Tricholoma album, $T$. malvacereum, $T$. portentosum, $T$. sejenctum and Tricholoma sp. were some of the species collectedonly from this site alone. The Simpson and Shannon's diversity index was observed to be 0.981 and 4.03 , respectively, while evenness and species richness was 2.248 and 0.55 , respectively. This high diversity of ectomycorrhizal diversity in Dachigam appears due to less human interference in this area as well as to the more availability of degradable materials. It was also noticed that huge plant litter accumulated in Dachigam forest floor may have helped to build up fertility and replenish the nutrients back into the soil.

Table.1 The ectomyorrhiza species collected from Zabarvan forest range in 2011, 2012 and 2013

\begin{tabular}{|c|c|c|c|c|}
\hline $\begin{array}{l}\text { Ectomycorrhizal } \\
\text { Species }\end{array}$ & Habitat & $\begin{array}{l}\text { Season } \\
\text { in } \\
\text { which it } \\
\text { noticed }\end{array}$ & $\begin{array}{l}\text { Site of } \\
\text { observation }\end{array}$ & $\begin{array}{l}\text { Altitude at } \\
\text { which } \\
\text { collected } \\
\text { (masl) }\end{array}$ \\
\hline $\begin{array}{l}\text { Agaricus } \\
\text { xanthoderma }\end{array}$ & $\begin{array}{l}\text { Mycorrhizal with Cedrus } \\
\text { deodara/ Pinus wallachiana }\end{array}$ & Spring & Dachigam & 2354 \\
\hline $\begin{array}{l}\text { Astraeus } \\
\text { hygometricus }\end{array}$ & $\begin{array}{l}\text { Mycorrhizal with } \\
P . \text { wallachiana/ C. deodara }\end{array}$ & Autumn & $\begin{array}{l}\text { Shankaracharia } \\
\text { Dachigam }\end{array}$ & $\begin{array}{l}2015 / \\
2189\end{array}$ \\
\hline Amanita ceciliae & $\begin{array}{l}\text { Mycorrhizal with } P \text {. } \\
\text { wallachiana }\end{array}$ & Spring & Dachigam & 2458 \\
\hline Amanita excelsea & $\begin{array}{l}\text { Mycorrhizal with } \\
\text { P. wallachiana }\end{array}$ & Autumn & Dachigam & 2369 \\
\hline Amanita inaurtia & $\begin{array}{l}\text { Mycorrhizal with } \\
\text { C. deodaral P. wallachiana }\end{array}$ & Autumn & Dachigam & 2487 \\
\hline $\begin{array}{l}\text { Boletus } \\
\text { subtomentosus }\end{array}$ & $\begin{array}{l}\text { Mycorrhizal with } \\
\text { C. deodara/ P. wallachiana }\end{array}$ & Autumn & $\begin{array}{l}\text { Dachigam } \\
\text { Shankaracharia }\end{array}$ & $2095 / 2019$ \\
\hline Boletus aereus & $\begin{array}{l}\text { Mycorrhizal with } \\
P . \text { wallachiana/ C. deodara }\end{array}$ & Autumn & Dachigam & 2687 \\
\hline Boletus cavipes & $\begin{array}{l}\text { Mycorrhizal with } \\
\text { C. deodara/ P. wallachiana }\end{array}$ & Autumn & Dachigam & 2596 \\
\hline Boletus gigas & $\begin{array}{l}\text { Mycorrhizal with } \\
P . \text { wallachiana }\end{array}$ & Autumn & Dachigam & 2658 \\
\hline $\begin{array}{l}\text { Cenoccoum } \\
\text { geophyllum }\end{array}$ & $\begin{array}{l}\text { Mycorrhizal with } \\
P . \text { wallachiana }\end{array}$ & Autumn & Dachigam & 2654 \\
\hline $\begin{array}{l}\text { Chroomphogus } \\
\text { tomentosus }\end{array}$ & $\begin{array}{l}\text { Mycorrhizal with } \\
\text { C. deodaral P. wallachiana }\end{array}$ & Spring & Dachigam & 2698 \\
\hline $\begin{array}{l}\text { Chroomphogus } \\
\text { vinicolor }\end{array}$ & $\begin{array}{l}\text { Mycorrhizal with } \\
\text { P. wallachiana }\end{array}$ & Spring & Dachigam & 2586 \\
\hline Entoloma sinatum & $\begin{array}{l}\text { Mycorrhizal with } \\
\text { C. deodara/ P. wallachiana }\end{array}$ & Autumn & Dachigam & 2478 \\
\hline Hebeloma & Mycorrhizal with & Autumn & Dachigam & 2612 \\
\hline
\end{tabular}




\begin{tabular}{|c|c|c|c|c|}
\hline crustuliniforme & P. wallachiana & & & \\
\hline $\begin{array}{l}\text { Hebeloma } \\
\text { cylindorosum }\end{array}$ & $\begin{array}{l}\text { Mycorrhizal with } \\
\text { C. deodara/ } P \text {. wallachiana }\end{array}$ & Autumn & Dachigam & 2489 \\
\hline $\begin{array}{l}\text { Hydnellum } \\
\text { aurantiacum }\end{array}$ & $\begin{array}{l}\text { Mycorrhizal with } C \text {. } \\
\text { deodara/ } P . \text { wallachiana }\end{array}$ & Spring & Dachigam & 2754 \\
\hline $\begin{array}{l}\text { Inocybe } \\
\text { appendiculata }\end{array}$ & $\begin{array}{l}\text { Mycorrhizal with } \\
\text { C. deodara/ P. wallachiana }\end{array}$ & $\begin{array}{l}\text { Summer } \\
\text { Autumn }\end{array}$ & $\begin{array}{l}\text { Dachigam } \\
\text { Shankaracharia }\end{array}$ & $2765 / 2345$ \\
\hline Inocybe fastigata & $\begin{array}{l}\text { Mycorrhizal with } \\
\text { P. wallachiana }\end{array}$ & $\begin{array}{l}\text { Spring, } \\
\text { Summer }\end{array}$ & $\begin{array}{l}\text { Shankaracharia } \\
\text { Shalimar }\end{array}$ & $2142 / 1987$ \\
\hline Inocybe geophylla & $\begin{array}{l}\text { Mycorrhizal with } \\
\text { C. deodara/ P. wallachiana }\end{array}$ & Spring & $\begin{array}{l}\text { Dachigam } \\
\text { Shankaracharia }\end{array}$ & $2698 / 2435$ \\
\hline Inocybe maculata & $\begin{array}{l}\text { Mycorrhizal with } \\
\text { C. deodaral P. wallachiana }\end{array}$ & Autumn & Shalimar & 2147 \\
\hline Laccaria bicolor & $\begin{array}{l}\text { Mycorrhizal with } \\
\text { C. deodaral } P \text {. wallachiana }\end{array}$ & Summer & $\begin{array}{l}\text { Dachigam } \\
\text { Shankaracharia }\end{array}$ & $2578 / 2256$ \\
\hline Laccaria laccata & Mycorrhizal with $C$. deodara & $\begin{array}{l}\text { Summer, } \\
\text { Autumn }\end{array}$ & $\begin{array}{l}\text { Dachigam } \\
\text { Shankaracharia }\end{array}$ & $2494 / 1998$ \\
\hline $\begin{array}{l}\text { Lactarius } \\
\text { controversus }\end{array}$ & $\begin{array}{l}\text { Mycorrhizal with } \\
\text { P. wallachiana }\end{array}$ & Autumn & $\begin{array}{l}\text { Shankaracharia } \\
\text { Shalimar }\end{array}$ & $1825 / 1894$ \\
\hline Lactarius delicious & $\begin{array}{l}\text { Mycorrhizal with } \\
\text { C. deodara/ } P \text {. wallachiana }\end{array}$ & Autumn & $\begin{array}{l}\text { Dachigam, } \\
\text { Shalimar }\end{array}$ & $2365 / 2134$ \\
\hline $\begin{array}{l}\text { Lactarius } \\
\text { pedicillatum }\end{array}$ & $\begin{array}{l}\text { Mycorrhizal with } \\
\text { C. deodara/ P. wallachiana }\end{array}$ & Autumn & $\begin{array}{l}\text { Dachigam } \\
\text { Shankaracharia }\end{array}$ & $2398 / 2032$ \\
\hline $\begin{array}{l}\text { Lycoperdon } \\
\text { pedicillatum }\end{array}$ & $\begin{array}{l}\text { Mycorrhizal with } \\
\text { C. deodara/ P. wallachiana }\end{array}$ & Autumn & $\begin{array}{l}\text { Dachigam } \\
\text { Shankaracharia }\end{array}$ & $2475 / 2258$ \\
\hline $\begin{array}{l}\text { Lycoperdon } \\
\text { perlatum }\end{array}$ & $\begin{array}{l}\text { Mycorrhizal with } \\
\text { C. deodaral } P \text {. wallachiana }\end{array}$ & Autumn & $\begin{array}{l}\text { Dachigam } \\
\text { Shankaracharia }\end{array}$ & $2457 / 2159$ \\
\hline $\begin{array}{l}\text { Lycoperdon } \\
\text { saccatum }\end{array}$ & $\begin{array}{l}\text { Mycorrhizal with } \\
\text { C. deodara/ } P \text {. wallachiana }\end{array}$ & Autumn & $\begin{array}{l}\text { Dachigam } \\
\text { Shankaracharia }\end{array}$ & $2467 / 2164$ \\
\hline $\begin{array}{l}\text { Lycoperdon } \\
\text { pyriforme }\end{array}$ & $\begin{array}{l}\text { Mycorrhizal with } \\
\text { C. deodaral } P \text {. wallachiana }\end{array}$ & Summer & $\begin{array}{l}\text { Dachigam, } \\
\text { Shalimar }\end{array}$ & $2787 / 2145$ \\
\hline $\begin{array}{l}\text { Macrolepiota } \\
\text { procera }\end{array}$ & $\begin{array}{l}\text { Mycorrhizal with } \\
\text { P. wallachiana }\end{array}$ & Autumn & $\begin{array}{l}\text { Dachigam, } \\
\text { Shalimar }\end{array}$ & $2658 / 2147$ \\
\hline $\begin{array}{l}\text { Macrolepoita } \\
\text { puellaris }\end{array}$ & $\begin{array}{l}\text { Mycorrhizal with } \\
\text { C. deodaral P. wallachiana }\end{array}$ & Autumn & $\begin{array}{l}\text { Dachigam, } \\
\text { Shalimar }\end{array}$ & $2475 / 2247$ \\
\hline Paxillus involutus & $\begin{array}{l}\text { Mycorrhizal with } \\
\text { P. wallachiana }\end{array}$ & Autumn & Dachigam & 2478 \\
\hline Pisolithus tinctorius & $\begin{array}{l}\text { Mycorrhizal with } \\
P \text {. wallachiana }\end{array}$ & Summer & Dachigam & 2158 \\
\hline Ramaria aurea & $\begin{array}{l}\text { Mycorrhizal with } \\
\text { C. deodara/ P. wallachiana }\end{array}$ & Autumn & Dachigam & 2245 \\
\hline Ramaria flaccid & $\begin{array}{l}\text { Mycorrhizal with } \\
\text { P. wallachiana }\end{array}$ & Autumn & $\begin{array}{l}\text { Dachigam } \\
\text { Shankaracharia }\end{array}$ & $2181 / 2097$ \\
\hline Ramaria flava & Mycorrhizal with & Autumn & Dachigam & 2215 \\
\hline
\end{tabular}




\begin{tabular}{|c|c|c|c|c|}
\hline & C. deodaral P. wallachiana & & & \\
\hline Ramaria formosa & $\begin{array}{l}\text { Mycorrhizal with } \\
\text { P. wallachiana }\end{array}$ & Autumn & Dachigam & 2104 \\
\hline Ramaria invalli & $\begin{array}{l}\text { Mycorrhizal with } \\
\text { C. deodara/ P. wallachiana }\end{array}$ & Autumn & Dachigam & 2369 \\
\hline Ramaria kuenzii & $\begin{array}{l}\text { Mycorrhizal with } \\
\text { C. deodara/ P. wallachiana }\end{array}$ & Spring & Dachigam & 2578 \\
\hline Rhizopogan roseulus & $\begin{array}{l}\text { Mycorrhizal with } \\
\text { C. deodara/ P. wallachiana }\end{array}$ & Spring & Dachigam & 2598 \\
\hline $\begin{array}{l}\text { Rhizopogan } \\
\text { vinicolor }\end{array}$ & $\begin{array}{l}\text { Mycorrhizal with } \\
\text { C. deodara/ P. wallachiana }\end{array}$ & Autumn & Dachigam & 2478 \\
\hline Rhizopogon vulgaris & $\begin{array}{l}\text { Mycorrhizal with } \\
\text { C. deodara/ P. wallachiana }\end{array}$ & Autumn & Dachigam & 2659 \\
\hline $\begin{array}{l}\text { Russula } \\
\text { atropurpurea }\end{array}$ & $\begin{array}{l}\text { Mycorrhizal with } \\
\text { C. deodaral P. wallachiana }\end{array}$ & Autumn & Dachigam & 2548 \\
\hline Russula brevipes & $\begin{array}{l}\text { Mycorrhizal with } \\
\text { C. deodara/ P. wallachiana }\end{array}$ & Autumn & Dachigam & 2487 \\
\hline Russula delica & $\begin{array}{l}\text { Mycorrhizal with } \\
\text { C. deodara/ P. wallachiana }\end{array}$ & Autumn & Dachigam & 2587 \\
\hline Russula densifolia & $\begin{array}{l}\text { Mycorrhizal with } \\
\text { C. deodaral P. wallachiana }\end{array}$ & Autumn & Dachigam & 2547 \\
\hline Russula emetica & Mycorrhizal with $C$. deodara & Autumn & Dachigam & 2014 \\
\hline Russula lilacea & $\begin{array}{l}\text { Mycorrhizal with } \\
\text { P. wallachiana }\end{array}$ & Autumn & Dachigam & 2016 \\
\hline Russula lutea & $\begin{array}{l}\text { Mycorrhizal with } \\
\text { C. deodaral P. wallachiana }\end{array}$ & Autumn & Dachigam & 2314 \\
\hline Russula paludosa & $\begin{array}{l}\text { Mycorrhizal with } \\
\text { C. deodara/ P. wallachiana }\end{array}$ & Autumn & Dachigam & 2366 \\
\hline Russula sanguinea & $\begin{array}{l}\text { Mycorrhizal with } \\
\text { C. deodaral P. wallachiana }\end{array}$ & Autumn & Dachigam & 2488 \\
\hline Russula sp. & $\begin{array}{l}\text { Mycorrhizal with } P \text {. } \\
\text { wallachiana }\end{array}$ & Autumn & Dachigam & 2115 \\
\hline Russula xerampelina & Mycorrhizal with $C$. deodara & Autumn & Dachigam & 2136 \\
\hline $\begin{array}{l}\text { Scleroderma } \\
\text { verrucosum }\end{array}$ & $\begin{array}{l}\text { Mycorrhizal with } \\
\text { C. deodara/ P. wallachiana }\end{array}$ & Autumn & Dachigam & 2147 \\
\hline Suillus cavipes & $\begin{array}{l}\text { Mycorrhizal with } \\
\text { P. wallachiana }\end{array}$ & $\begin{array}{l}\text { Summer } \\
\text { Autumn }\end{array}$ & Dachigam & 2132 \\
\hline Suillus granulates & $\begin{array}{l}\text { Mycorrhizal with } \\
\text { C. deodaral P. wallachiana }\end{array}$ & Summer & Dachigam & 2373 \\
\hline Suillus luteus & $\begin{array}{l}\text { Mycorrhizal with } \\
\text { C. deodaral } P \text {. wallachiana }\end{array}$ & Summer & Dachigam & 2347 \\
\hline Suillus placidus & $\begin{array}{l}\text { Mycorrhizal with } \\
\text { C. deodara/ P. wallachiana }\end{array}$ & Summer & $\begin{array}{l}\text { Dachigam } \\
\text { Shankaracharia }\end{array}$ & $2365 / 2259$ \\
\hline Thelephora terrestris & Mycorrhizal with & Autumn & Dachigam & $2345 / 2236$ \\
\hline
\end{tabular}




\begin{tabular}{|c|c|c|c|c|}
\hline & C. deodaral P. wallachiana & & Shankaracharia & \\
\hline Tricholoma terreum & $\begin{array}{l}\text { Mycorrhizal with } \\
\text { C. deodara/ } P \text {. wallachiana }\end{array}$ & Autumn & $\begin{array}{l}\text { Dachigam } \\
\text { Shankaracharia }\end{array}$ & $2317 / 2247$ \\
\hline Tricholoma album & $\begin{array}{l}\text { Mycorrhizal with } \\
\text { C. deodaral P. wallachiana }\end{array}$ & Autumn & Dachigam & 2479 \\
\hline $\begin{array}{l}\text { Tricholoma } \\
\text { malvacereum }\end{array}$ & $\begin{array}{l}\text { Mycorrhizal with } \\
\text { C. deodara/ P. wallachiana }\end{array}$ & Autumn & Dachigam & 2147 \\
\hline $\begin{array}{l}\text { Tricholoma } \\
\text { portentosum }\end{array}$ & $\begin{array}{l}\text { Mycorrhizal with } \\
\text { C. deodaral } P \text {. wallachiana }\end{array}$ & Autumn & Dachigam & 2689 \\
\hline $\begin{array}{l}\text { Tricholoma } \\
\text { scalpturattum }\end{array}$ & $\begin{array}{l}\text { Mycorrhizal with } \\
\text { C. deodara/ P. wallachiana }\end{array}$ & Autumn & Dachigam & 2789 \\
\hline $\begin{array}{l}\text { Tricholoma } \\
\text { sejenctum }\end{array}$ & $\begin{array}{l}\text { Mycorrhizal with } \\
\text { C. deodaral P. wallachiana }\end{array}$ & Autumn & Dachigam & 2896 \\
\hline Tricholoma sp. & $\begin{array}{l}\text { Mycorrhizal with } \\
\text { C. deodaral } P \text {. wallachiana }\end{array}$ & Autumn & Dachigam & 2687 \\
\hline Tuber sp. & $\begin{array}{l}\text { Mycorrhizal with } \\
\text { P. wallachiana }\end{array}$ & Autumn & Shankaracharia & 2,015 \\
\hline
\end{tabular}

Table.2 Seasonal distribution of ectomycorrhizal species (No.) observed in Zabarvan forest range (2011-2013)

\begin{tabular}{|l|c|c|c|c|}
\hline Location & \multicolumn{2}{|c|}{ Spring season Summer season } & Autumn season & $\begin{array}{c}\text { Overall species } \\
\text { noticed }\end{array}$ \\
\hline Dachigam & 08 & 08 & 49 & 65 \\
\hline Shalimar & 02 & 02 & 07 & 11 \\
\hline Shankaracharia & 02 & 05 & 15 & 25 \\
\hline Total & 09 & 11 & 45 & 67 \\
\hline
\end{tabular}

Fig.1 Zabarvan forest range in North Western Himalaya

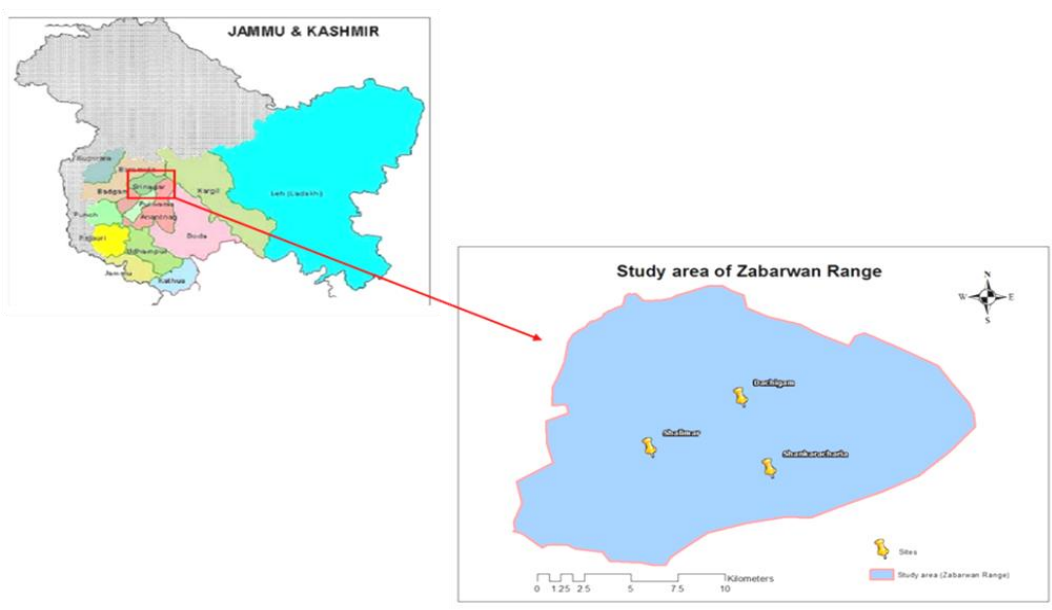


Table.3 Species- and family-wise distribution of ectomycorrhizal fungi observed in Zabarvan forest range

\begin{tabular}{|c|c|c|c|}
\hline $\begin{array}{l}\text { S. } \\
\text { No. }\end{array}$ & Family & Species & Total \\
\hline 01 & Agaricacea & $\begin{array}{l}\text { Agaricus xanthoderma, Lycoperdon pedicillatum, L. perlatum, } L \text {. } \\
\text { pyriforme, L. saccatum }\end{array}$ & 5 \\
\hline $\mathbf{0 2}$ & Diplocystidiaceae & Astraeus hygometricus & 1 \\
\hline 03 & Amanitaceae & Amanita ceciliae, A. excelsea, A. inaurtia & 3 \\
\hline 04 & Boletaceae & Boletus subtomentosus, B. aereus, B. cavipes, B. gigas & 4 \\
\hline 06 & Gloniaceae & Cenoccoum geophyllum & 1 \\
\hline 07 & Gomphidiaceae & Chroomphogus tomentosus, $C$. vinicolor & 2 \\
\hline 08 & Entolomataceae & Entoloma sinatum & 1 \\
\hline 09 & Hymeogastraceae & Hebeloma crustuliniforme, H. cylindorosum & 2 \\
\hline 10 & Bankeraceae & Hydnellum aurantiacum & 1 \\
\hline 11 & Inocybaceae & Inocybe appendiculata, I. fastigata, I. geophylla, I. maculate & 4 \\
\hline 12 & Hydnangiaceae & Laccaria bicolor, Laccaria laccata & 2 \\
\hline 13 & Russulaceae & $\begin{array}{l}\text { Lactarius controversus, } L . \text { delicious, L. pedicillatum, Russula } \\
\text { atropurpurea, } R . \text { brevipes, } R . \text { delica, } R . \text { densifolia, } R . \text { emetica, } R . \\
\text { lilacea, } R . \text { lutea, R. paludosa, R. sanguinea, R. xerampelina, } \\
\text { Russula sp. }\end{array}$ & 14 \\
\hline 14 & Lepiotaceae & Macrolepiota procera, M. puellaris & 2 \\
\hline 15 & Paxillaceae & Paxillus involutus & 1 \\
\hline 16 & Sclerodermataceae & Pisolithus tinctorius, Scleroderma verrucosum & 2 \\
\hline 18 & Gomphaceae & $\begin{array}{l}\text { Ramaria aurea, } R \text {. flaccid, } R \text {. flava, } R \text {. formosa, } R \text {. invalli, } R \text {. } \\
\text { kuenzii }\end{array}$ & 6 \\
\hline 19 & Rhizopogonaceae & Rhizopogan roseulus, $R$. vinicolor, $R$. vulgaris & 3 \\
\hline 20 & Suillaceae & Suillus cavipes, S. granulates, S. luteus, S. placidus & 4 \\
\hline 21 & Thelephoraceae & Thelephora terrestris & 1 \\
\hline 22 & Tricholomataceae & $\begin{array}{l}\text { Tricholoma terreum, T. album, T. malvacereum, } T \text {. portentosum, } \\
\text { T. scalpturattum, T. sejenctum, Tricholoma sp. }\end{array}$ & 7 \\
\hline 23 & Tubaraceae & Tuber sp. & 1 \\
\hline
\end{tabular}

Table.4 Species diversity, richness and evenness of ectomycorrhiza in Zabarvan forest range of Kashmir

\begin{tabular}{|c|c|c|c|}
\hline & Dachigam & Shalimar & Shankaracharia \\
\hline No. of species & 65 & 11 & 25 \\
\hline Total No. of individuals & 1502 & 125 & 294 \\
\hline Simpson diversity index (1-D) & 0.981 & 0.910 & 0.939 \\
\hline Shannon diversity index (H) & 4.03 & 2.36 & 2.903 \\
\hline Evenness (En) & 2.248 & 2.26 & 2.195 \\
\hline Spieces richness (s) & 0.55 & 0.19 & 0.40 \\
\hline
\end{tabular}


Amongst the 21 species collected from Shankaracharia forest area, Astraeus hygometricus and Tuber sp. were two species collected only at this site. The Simpson and Shannon's diversity index was 0.939 and 2.903, respectively, similarly evenness and species richness and was found to be 2.195 and 0.40 respectively, was found low compared to Dachigam forest range. This may due to the anthropogenic activity. Similarly, 11 species were collected from Shalimar site. The Simpson and Shannon's diversity index was 0.91 and 2.36, respectively, while evenness and species richness and was found to be 2.26 and 0.19 respectively, was found lowest compared to Dachigam forest range and shankaracharia forest range. This may due to the interference of human activities and more so various tourist places have come up in this area. These results are in partial conformity with Bhatt (1986) and Adhikari (1999), in Dachigam, diversity index was high because it is legally protected and has less anthropogenic activity. Season-wise more diverse fungi were observed in Dachigam and low diversity index in Shalimar region.

It can be concluded Since the mychorrhiza play an important role to maintain the health of forests besides their medicinal importance and nutritional value in most of the cases, therefore it becomes quite necessary to explore, document and conserve this natural wealth.

\section{Acknowledgement}

The authors are thankful to all the individuals directly and indirectly assisting in the sample collection and laboratory preparations throughout the study.

\section{References}

Adhikari, M.K., 1999. Mushrooms of Nepal (ed. Durrieu, G.)

Agerer, R., 2006. Fungal relationships and structural identity of their ectomycorrhizae. Mycol Progress 5(2):
67-107.

Auc ina, A., Rudawska, M., Leski, T., Skridaila, A., Riepšas, E and Iwanski, M. 2007. Growth and mycorrhizal community structure of Pinus sylvestris seedlings following the addition of forest. Applied and Environmental Microbiology73: 4867-4873.

Batra, R. and Batra S.W.T. 1963. Indian Discomycetes. University of Kansa Science Bulletin, 44: 109-256.

Beig, M.A., Dar, G. H., Ganai N. A. and Qazi N. A. 2008. Some hitherto unreported macrofungi from India. Journal of Mycology and Plant Pathology38: 208210.

Bhatt, R.P., 1986. Systematics and ecobiology of some agaric family. $\mathrm{Ph} \mathrm{D}$ thesis, Himachal Pradesh, University, SimlaIndia.

Carlson, K.M., Asner, G.P., Flint, H.R., Ostertag R. and Martin. R. E. 2007. Hyperspectral remote sensing of canopy biodiversity in Hawaiian lowland rainforests. Ecosystems 10: 536- 549.

Cooke, M.C., 1870. Answers to correspondents. Gardener's Chronicle15: 605.

Dar, G.H., Beig M. A. and Ganai. N. A. 2009. Diversity of ectomycorrhizal fungi of various forests in district Baramulla (Jammu \& Kashmir). Indian Journal of Forestry32: 137-140.

Deroman, M., Clavaria V. and Demiguel. A. M. 2005. A revision of the description of ectomycorrhiza published since 1961. Mycol Res 109: 1063-1104.

Engola, A.P.O., Eilu, G., Kabasa, J. D., Kisovi, L., Munishi P. K. T. and Olila. D. 2007. Ecology of edible indigenous mushrooms of the Lake Victoria basin (Uganda). Research Journal of Biological Sciences 2(1): 62-68.

Economic survey J \& K. 2013 -14. Directorate of Economic \& Statistics, page no: 242.

FSI, State of the forest report. 2013. Forest Survey of India, Ministry of Environment and Forests, Government 
of India, Dehradun.

Gaston, K.J., 2000. Global patterns in biodiversity. Nature 405: 220-227.

Hawksworth, D.L., 2004. Fungal diversity and its implications for genetic resource collections. Studies in Mycology 50: 917.

Kaul, T.N., and Kachroo. J.L., 1974. Common edible mushrooms of Jammu and Kashmir. Journal of the Bombay Natural History Society71: 26-31.

Kaul, T.N., Kachroo J. L. and Raina, A. 1978. Common edible mushrooms of Jammu and Kashmir. Indian Mushroom Science1: 517-529.

Kirk, P.M., Cannon, P. F., David J. C. and Stalpers. J. A. 2001. Ainsworth \& Bisby's Dictionary of the Fungi. 9th edn. CAB International Publishing, Kew, Surrey, Commonwealth Mycological Institute, UK, 655p.

Kornerup, A., and Wanscher, J., H. 1978. Methuen handbook of Colour. $3^{\text {rd }} \mathrm{Ed}$. Methuen and Co., Ltd., London, pp: 243.

Kuo, M. 2001. Making Spore Prints (www.bluewillopages.com/mushroomex pert/ herbarium.html).

Kuo, M,. 2004. Agrocybe molesta (www.mushroomexpert.com/ agrocybe_molesta. html).

Manoharachary, C., Sridhar, K., Singh, R. A., Suryanarayanan, T. S., Rawat S. and Johri. B. N. 2005. Fungal biodiversity: Distribution, conservation and prospecting of fungi from India. Current Science, 89: 58-71.

Margalef, R., 2008. Correspondence between the classic types of lakes and the structural and dynamic properties of their population. Verh. Int. Ver. Theor. Angew. Limnol., 15: 169-170.

Mihali, I., 1995. Abundancia a distribúcia plodníc húb(prebierkovej [Abundance and distribution of fruiting bodies of fungi in terms beech]. v podmienkach prebierkovej buciny. Forestry 41: 218 223.

Murrill, W.A., 1924. The Boletaceae of North
America-1. Mycologia 1: 4-18.

Odum, E.P., 1971. Fundamentals of ecology, $3^{\text {rd }}$ edition, WB Saunders, Phildelphia PA.

Ohenojo, E,. 1993. Effect of Weather Condition on the Larger Fungi at Different Forest Sites in the Northern Finland in 19761988. Vol. A243. Acvta University, Oulu, Finland, p. 69.

Packham, J.M., May, T. M., Brown, M. J., Wardlaw T. J. and Mills. K. A. 2002. Macrofungal diversity and community ecology in mature and regrowth wet eucalypt forest in Tasmania: A multivariate study. Australian Ecology 27: 149-161.

Pielou, E.C,. 1996. The measurement of diversity in different types of biological collections. J. Theor. Biol., 13: 131-144.

Read, D. J., Leake J. R. and Perez-Morno, J. 2003. Mycorrhizal fungi as drivers of ecosystem processes in heathland and boreal forest biomes. Canadian Journal of Botany82: 1243-1263.

Reddy, C., Dutta S. K. and Jha. C. S. 2013. Analysing the gross and net deforestation rates in India. Curr Sci105(11), 1492.

Rodgers, W.A., Panwar, H. S., Mathur, V. B. 2002. Wildlife Protected Area Network in India: A Review (Executive Summary). Wildlife Institute of India, Dehardun.

Samant, S.S. and Dhar, U. 1997. Diversity, endemism and economic potential of wild edible plants of Indian Himalayas. International Journal of Sustainable Development and Wild Ecology4: 179191.

Simpson, E.H., 1949. Measurement of diversity. Nature 163, 688.cited in Magurran, A.E., 2004, Measuring biological diversity, Blackwell Publishing: Oxford, UK., pp: 256.

Sheikh, P.A., Dar, G. H., Beig M. A. and Kousar. S. 2014. Two hitherto unreported macrofungi from zabarvan range of Kashmir Himalaya, India. Caribbean Journal of Science and Technology 2: 399-404. 
Tedersoo, L., and Smith. M.E., 2013. Lineages of ectomycorrhizal fungi revisited: foraging strategies and novel lineages revealed by sequences from belowground. Fungal Biol. Rev., 27, 83-99.

Vishwakarma, M.P. 2010. Systematics and Ecological Studies on Agaricaceae and Coprinaceae of Garhwal Himalaya. M.Phil. thesis, HNB Garhwal University (A Central University), Srinagar, Garhwal, Uttarakhand, India.

Vishwakarma, M.P., Bhatt, R. P and Gairola, S. 2011. Some medicinal mushrooms of Garhwal Himalaya, Uttarakhand, India. International Journal of Medicinal and Aromatic Plants. 1: 33-40.

Walting, R., and Gregory. N.M. 1980. Larger fungi from Kashmir. Nova Hedwigia, 32: 494-564.

Watling, R., and Abraham. S.P. 1992.
Ectomycorrhizal fungi of Kashmir forests. Mycorrhiza. 2: 81-87.

Wood- eggenschwiler, S. and Barlocher, F. 1985. Geographical distribution of Ingoldian fungi, Verhandlungen der international Vereinigung Limnologie, 22: 2780- 2785.

Young, H.E., 1940. Mycorrhiza and growth of Pinus and Araucaria: The influence of different species of mycorrhizae forming fungi on seedling growth. Journal of Australian Agriculture Science 6: 21-25.

Zak, J., 1969. Fungal communities of desert ecosystems: Links to climate change. p. 659-682. In: The Fungal Community (Eds. J. Dighton, J.F. White and P. Oudemans). Taylor and Francis, Boca Raton, USA.

\section{How to cite this article:}

Sheikh, P.A., Baby Summuna, G.H. Dar, Sajad-Un-Nabi and Khurshid Ahmad Mir. 2019. Ectomycorrhizal Diversity in Zabarvan Forest Range of North Western Himalaya. Int.J.Curr.Microbiol.App.Sci. 8(05): 2312-2323. doi: https://doi.org/10.20546/ijcmas.2019.805.273 